\title{
Negative myoclonus associated with pregabalin
}

\author{
Kwan-Do Park, Min-Ku Kim, Se-Jin Lee \\ Department of Neurology, Yeungnam University College of Medicine, Daegu, Korea
}

Negative myoclonus (NM) is a jerky, shock-like involuntary movement caused by a sudden, brief interruption of muscle contraction. An 80-year-old man presented with multifocal NM and confusion. Two days before the onset of NM, he commenced the intake of pregabalin at a dose of $150 \mathrm{mg} /$ day for neuropathic pain. His NM resolved completely and mental status improved gradually after the administration of lorazepam intravenously and the discontinuation of pregabalin. Our study suggests that pregabalin can cause NM even in patients without a history of seizures.

Keywords: Myoclonus; Negative myoclonus; Pregabalin

\section{INTRODUCTION}

Myoclonus is a brief involuntary jerky movement of a muscle or a group of muscles. Myoclonus is classified as positive and negative myoclonus (NM) [1,2]. Positive myoclonus is characterized by sudden bursts of muscular activity, which cause shock-like muscle contractions and subsequent relaxation. $\mathrm{NM}$ is caused by a sudden and brief interruption of muscle contraction, which can lead to sudden postural lapses and dropping of objects from hands with difficulties in writing and feeding, head nodding, or, at times, gait instability and falls.

Pregabalin (PGB) is a new anticonvulsant approved not only as adjunctive therapy for the management of focal epilepsy, but also for the treatment of neuropathic pain, neuralgia, and anxiety disorders. Thus, there has been a significant increase in the volume of prescriptions of PGB. PGB is structurally similar to the neurotransmitter gamma-aminobutyric acid

Received: March 20, 2018, Revised: May 17, 2018

Accepted: May 31, 2018

Corresponding Author: Se-Jin Lee, Department of Neurology, Yeungnam University College of Medicine, 170, Hyeonchung-ro, Nam-gu, Daegu 42415, Korea Tel: +82-53-620-3683, Fax: +82-53-627-1688

E-mail: sejinmayo@ynu.ac.kr
(GABA); however, it does not act on the GABA receptors and transporters. The major mechanism of action is via inhibiting the release of excitatory neurotransmitters by binding to the $\alpha 2-\delta-1$ protein, which is a membrane protein subunit of voltage-sensitive calcium channels. Reportedly, PGB is known to elicit positive and NM. However, to date, NM has never been described in Korea [3-8]. We report a case of PGB-induced NM.

\section{CASE}

An 80-year-old man presented with sudden new onset of unsteadiness of gait, which led to falls and dropping of arms downward when his arm was raised forward. He reported no abnormality/difficulty before going to bed the night prior to presentation. However, that night, while walking to the washroom, he experienced a loss of power in his knees, and his legs collapsed. He reported no loss of consciousness and could stand up by himself immediately after the incident. When he attempted to stretch his arms straight forward, he could not maintain that posture. However, he could raise his arms again soon thereafter. This symptom recurred sporadically but did not occur when he was lying down or sitting with his arms on the ground. Although he had no history

Copyright (C) 2018 Yeungnam University College of Medicine

This is an Open Access article distributed under the terms of the Creative Commons Attribution Non-Commercial License (http://creativecommons.org/licenses/by-nc/4.0/) which permits unrestricted non-commercial use, distribution, and reproduction in any medium, provided the original work is properly cited. 
of cognitive impairment, he was observed to be dull and developed memory impairment and confusion.

He reported the intake of $150 \mathrm{mg}$ of PGB and $200 \mathrm{mg}$ of celecoxib for neuropathic pain, 2 days before presentation. He had been treated regularly for diabetes and hypertension for 10 years, and the regimen had not been changed recently. Eight years ago, he underwent colectomy for colon cancer. He reported no personal or family history of seizure or myoclonus.

At the time of his hospital visit, his vital signs showed a blood pressure of $140 / 80 \mathrm{mmHg}$, pulse rate of 92 beats/min, and body temperature of $36.7^{\circ} \mathrm{C}$. Upon neurological examination, he was alert and explained his symptoms but appeared to be disoriented to time and place and was confused about his illness. When he stretched both arms forward with dorsiflexion at the wrists in the sitting position, NM was observed irregularly, although NM was not observed at rest and was not provoked by tactile stimuli. He showed no other focal neurological deficits except bilateral segmental sensory impairment in the area innervated by the fifth lumbar and the first sacral nerve roots.

Laboratory tests showed decreased hemoglobin $11.5 \mathrm{~g} / \mathrm{dL}$, (range, 14-18 g/dL) and glomerular filtration rate (GFR) 47.6 $\mathrm{mL} / \mathrm{min}$, and an increase in glycated hemoglobin $7.9 \%$ (range, 4.4-6.3\%), and serum concentration of blood urea nitrogen (BUN) $29.5 \mathrm{mg} / \mathrm{dL}$ (range, 8-23 mg/dL). However, his serum creatinine, ammonia, electrolytes, liver and thyroid function tests were within the reference ranges. Three days after admission, his serum concentrations of creatinine $0.98 \mathrm{mg} / \mathrm{dL}$ (range, $0.5-1.5 \mathrm{mg} / \mathrm{dL}$ ) and BUN $10.9 \mathrm{mg} / \mathrm{dL}$ (range, $8-23$ $\mathrm{mg} / \mathrm{dL}$ ), as well as GFR $83.4 \mathrm{~mL} / \mathrm{min}$ showed improvement.

We diagnosed him with PGB-induced NM and administered $4 \mathrm{mg}$ of lorazepam intravenously and discontinued the administration of $\mathrm{PGB}$, which led to complete disappearance of his NM. Electroencephalography (EEG) performed 5 hours later showed excessive low amplitude beta waves but no epileptiform discharges. Brain computed tomography showed diffuse brain atrophy and degenerative changes, and he refused to undergo brain magnetic resonance imaging. After admission, he received fluid therapy but no anticonvulsant. The following day his mental status improved gradually, and on day 4 of hospitalization, he was alert and oriented and was discharged. No recurrence of NM has been observed over 3 years.

\section{DISCUSSION}

Reportedly, dizziness, somnolence, ataxia, tremor, weight gain, dose-dependent myoclonus, cortical-NM, and generalized myoclonic status epilepticus are known adverse effects of PGB. Several reports have described the occurrence of myoclonus associated with PGB use. Huppertz et al. [3] have reported four cases of focal positive myoclonus, Heckmann et al. have described a case of asterixis [4], Knake et al. have described a case of myoclonic status epilepticus [5], Hellwig et al. have described a case of cortical-NM[6], and Kim et al. have described nine cases of NM [7]. Our patient did not report history of NM or epilepsy. We diagnosed him with PGB-induced NM because he developed NM and encephalopathy 2 days after the intake of PGB, and his symptoms disappeared completely after the administration of lorazepam intravenously and the discontinuation of PGB. Thereafter, symptoms of encephalopathy also improved gradually, and NM did not recur over 3 years.

$\mathrm{NM}$ occurs in patients during certain specific postures but does not occur at rest [1,2]. If NM occurs in the axial or leg muscles, gait and stance may become unsteady with repetitive loss of muscle tone, which leads to falls. In our patient, NM did not occur at rest but appeared when the patient raised his arms forward or held his neck in an upright position, and positive myoclonus was not observed. Positive myoclonus can be easily diagnosed; however, NM usually shows a subtle presentation and disappears with the withdrawal of the causative agent. Thus, a significant number of patients may not visit a physician/clinic because the symptoms might be missed. Furthermore, patients with NM may be clinically misdiagnosed with a tremor, weakness, or simple falls, which leads to underdiagnosis of this condition and consequent underestimation of the incidence of NM [7].

No studies have reported the association between the dosage, duration of use, and serum concentration of PGB and the occurrence of NM. The occurrence of NM after the introduction of PGB supports the previous observations of Huppertz et al. [3], who described a threshold phenomenon rather than linear dose dependency. Kim et al. [7] reported that the mean dosage of PGB administered was $217 \mathrm{mg} /$ day (in five, $150 \mathrm{mg}$; in four, $300 \mathrm{mg}$ ) and the mean time lapse until the development of $\mathrm{NM}$ after the administration of PGB was 3.7 days. These results were in agreement with previous 
reports $[4,6,8]$. Therapeutic drug monitoring for PGB is not performed in routine clinical practice; therefore, data pertaining to the serum concentrations of PGB are not available. Therefore, we could not measure the serum concentrations of PGB.

Based on reports in the literature, antipsychotics, antiparkinsonian medications, anesthetics, anti-infective drugs, opiates, and antidepressants are all known to be associated with the occurrence of myoclonus [9]. If a patient is diagnosed with $\mathrm{NM}$, recent medication history should be thoroughly investigated. Furthermore, it is important to perform complete laboratory examination to identify a toxic or metabolic disorder, which could cause NM.

Most patients with PGB-induced NM described by previous studies have shown normal renal function [7]. However, patients with diminished renal function are at a high risk of developing myoclonus. PGB is excreted unchanged via the kidneys; thus, patients with impaired renal function tend to show elevated serum concentrations of PGB, and excessive plasma accumulation of the drug may lead to accumulation in the cerebrospinal fluid with consequent neurotoxicity [8]. This observation is supported by the complete resolution of symptoms after dialysis [8]. Therefore, drug intoxication secondary to impaired renal excretion has been proposed as a possible underlying mechanism in the development of myoclonus. However, the pathophysiological mechanism that underlies the development of PGB-induced NM remains unknown. With regard to positive myoclonus, it has been suggested that the serotonin neurotransmitter system plays a role in the development of gabapentin-induced myoclonus [10-12]. PGB and gabapentin have a similar chemical structure and show the same mechanism of action. Therefore, we speculate that PGB increases the serum concentration of serotonin, which consequently can cause NM.

In our patient, the initial serum concentrations of creatinine, BUN, and GFR were $1.37 \mathrm{mg} / \mathrm{dL}, 29.5 \mathrm{mg} / \mathrm{dL}$, and 47.6 $\mathrm{mL} / \mathrm{min}$, respectively. Three days after admission, serum concentrations of creatinine, BUN, and GFR improved to 0.98 $\mathrm{mg} / \mathrm{dL}, 10.9 \mathrm{mg} / \mathrm{dL}$, and $83.4 \mathrm{~mL} / \mathrm{min}$, respectively. Initial serum concentration of BUN was slightly increased but not to the level which can cause NM. After administration of fluid therapy, the patient's renal function improved. Therefore, it can be hypothesized that he had already presented with slightly impaired renal function, which caused an elevation of his serum concentration of PGB and eventually caused NM and encephalopathy.

NM in toxic metabolic encephalopathies, i.e., "asterixis," which displays the characteristics of subcortical NM. However, in a few cases, the demonstration of a cortical correlate using an averaging technique of the electromyographic-EEG signals $[1,2]$. Our patient did not report history of epile- psy, and NM was bilaterally symmetrical and multifocal in his neck and arms and was not provoked by tactile stimuli. Thus, we propose that NM observed in our patient was aste- rixis associated with PGB intoxication.

In conclusion, PGB can cause NM and also provoke positive myoclonus. In patients diagnosed with PGB-induced $\mathrm{NM}$, physicians should immediately discontinue the administration of PGB and administer clonazepam.

\section{CONFLICT OF INTEREST}

No potential conflict of interest relevant to this article were reported.

\section{ORCID}

Kwan-Do Park, https://orcid.org/0000-0003-0485-2705

Se-Jin Lee, https://orcid.org/0000-0001-9391-0086

\section{REFERENCES}

1. Rubboli G, Tassinari CA. Negative myoclonus. An overview of its clinical features, pathophysiological mechanisms, and management. Neurophysiol Clin 2006;36:337-43.

2. Eberhardt O, Topka H. Myoclonic disorders. Brain Sci 2017; 7. pii: E103.

3. Huppertz HJ, Feuerstein TJ, Schulze-Bonhage A. Myoclonus in epilepsy patients with anticonvulsive add-on therapy with pregabalin. Epilepsia 2001;42:790-2.

4. Heckmann JG, Ulrich K, Dütsch M, Neundörfer B. Pregabalin associated asterixis. Am J Phys Med Rehabil 2005;84:724.

5. Knake S, Klein KM, Hattemer K, Wellek A, Oertel WH, Hamer $\mathrm{HM}$, et al. Pregabalin-induced generalized myoclonic status epilepticus in patients with chronic pain. Epilepsy Behav 2007; 11:471-3.

6. Hellwig S, Amtage F. Pregabalin-induced cortical negative myoclonus in a patient with neuropathic pain. Epilepsy Behav 2008;13:418-20.

7. Kim JB, Jung JM, Park MH, Lee EJ, Kwon DY. Negative myoclonus induced by gabapentin and pregabalin: a case series and systematic literature review. J Neurol Sci 2017;382: 36-9. 
8. Healy DG, Ingle GT, Brown P. Pregabalin- and gabapentinassociated myoclonus in a patient with chronic renal failure. Mov Disord 2009;24:2028-9.

9. Brefel-Courbon C, Gardette V, Ory F, Montastruc JL. Druginduced myoclonus: a French pharmacovigilance database study. Neurophysiol Clin 2006;36:333-6.

10. Rao ML, Clarenbach P, Vahlensieck M, Krätzschmar S. Gabapentin augments whole blood serotonin in healthy young men.
J Neural Transm 1988;73:129-34.

11. Pappert EJ, Goetz CG, Stebbins GT, Belden M, Carvey PM. 5-Hydroxytryptophan-induced myoclonus in guinea pigs: mediation through 5-HT1/2 receptor subtypes. Eur J Pharmacol 1998;347:51-6.

12. Asconapé J, Diedrich A, DellaBadia J. Myoclonus associated with the use of gabapentin. Epilepsia 2000;41:479-81. 